Л. Л. Рыскина, Л. А. Жидова. Дифференциальное уравнение типа Клеро в частных производных со степенной функцией

УДК 517.952

DOI: $10.18101 / 2304-5728-2019-1-41-48$

\title{
ДИФФЕРЕНЦИАЛЬНОЕ УРАВНЕНИЕ ТИПА КЛЕРО В ЧАСТНЫХ ПРОИЗВОДНЫХ СО СТЕПЕННОЙ ФУНКЦИЕЙ
}

\author{
(C) Рыскина Лилия Леонидовна \\ кандидат физико-математических наук, доцент, \\ Томский государственный педагогический университет \\ Россия, 634061, г. Томск, ул. Киевская, 60, \\ E-mail: ryskina@tspu.edu.ru \\ (C) Жидова Любовь Александровна \\ кандидат педагогических наук, доцент, \\ Томский государственный педагогический университет \\ Россия, 634061, г. Томск, ул. Киевская, 60, \\ E-mail: gidovala@yandex.ru
}

Целью исследования данной работы является изучение дифференциальных уравнений типа Клеро в частных производных первого порядка со специальной правой частью. Для обыкновенного дифференциального уравнения Клеро нахождение общего решения не представляет особого труда и подробно описано в курсах теории обыкновенных дифференциальных уравнений. Помимо общего решения, представляющего собой семейство интегральных прямых, для обыкновенного дифференциального уравнения Клеро существует особое решение, которое есть огибающая данного семейства. В теории дифференциальных уравнений в частных производных существуют дифференциальные уравнения типа Клеро, которые представляют собой многомерные обобщения обыкновенного дифференциального уравнения Клеро. Отметим, что для уравнения в частных производных типа Клеро не всегда существует особое решение. Настоящая статья посвящена проблеме описания особого решения уравнений типа Клеро, правая часть которой имеет вид степенной функции от произведения n-сомножителей.

Ключевые слова: дифференциальные уравнения в частных производных; дифференциальные уравнения типа Клеро; особые решения; степенная функция.

Для цитирования:

Рыскина Л. Л., Жидова Л. А. Дифференциальное уравнение типа Клеро в частных производных со степенной функцией // Вестник Бурятского государственного университета. Математика, информатика. 2019. № 1. С. 41-48.

\section{Введение}

Предлагаемая вниманию читателя научная статья посвящена изучению дифференциальных уравнений в частных производных типа Клеро. Класс этих уравнений широко известен и подробно описан в курсах теории обыкновенных дифференциальных уравнений [1-3] и теории дифферен- 
циальных уравнений в частных производных [4-6]. Основные определения и алгоритм получения общего решения приведен нами для описания концептуальных положений теории. Общее решение дифференциальных уравнений типа Клеро в частных производных представляет собой семейство линейных функций, однако поиск особых решений уравнений типа Клеро привлекает внимание исследователей, так как до сих пор нет единого алгоритма их получения. В аспекте проблематики нашего исследования представляют интерес следующие работы [7-10]. Проведенные в них исследования внесли заметный вклад в развитие подходов к поиску особых решений уравнений типа Клеро. По-прежнему актуальной остается проблема поиска особого решения для конкретных функций. Настоящая статья посвящена проблеме нахождения особого решения уравнений типа Клеро в частных производных, правая часть которого имеет вид степенной функции от произведения n-независимых переменных.

\section{1 Общие сведения}

В теории дифференциальных уравнений в частных производных изучают уравнения вида:

$$
y-\sum_{i=1}^{n} \frac{\partial y}{\partial x_{i}} x_{i}=\psi\left(\frac{\partial y}{\partial x_{1}}, \frac{\partial y}{\partial x_{2}}, \ldots, \frac{\partial y}{\partial x_{n}}\right),
$$

где $y=y\left(x_{1}, x_{2}, \ldots, x_{n}\right)$. Уравнения вида (1) называют уравнениями типа Клеро [4-6] в частных производных. Сделаем замену $\frac{\partial y}{\partial x_{i}}=z_{i}(x)$, $i=1,2, \ldots, n$. Тогда уравнение (1) в новых обозначениях примет вид:

$$
y=\sum_{i=1}^{n} z_{i} x_{i}+\psi\left(z_{1}, z_{2}, \ldots, z_{n}\right)
$$

Продифференцируем уравнение (2) по переменным $x_{i}, i=1,2, \ldots, n$ :

$$
\sum_{j=1}^{n}\left(\frac{\partial z_{j}}{\partial x_{i}} \cdot x^{j}+z_{j} \cdot \frac{\partial x_{j}}{\partial x_{i}}+\frac{\partial \psi}{\partial z_{j}} \frac{\partial z_{j}}{\partial x_{i}}\right)=z_{i} .
$$

Учтем, что $\frac{\partial x_{j}}{\partial x_{i}}=\delta_{i j}$, где $\delta_{i j}-$ символ Кронекера и $\sum_{j=1}^{n} z_{j} \delta_{i j}=z_{i}$,

$$
\sum_{j=1}^{n} \frac{\partial z_{j}}{\partial x_{i}}\left(x_{j}+\frac{\partial \psi}{\partial z_{j}}\right)+z_{i}=z_{i} .
$$

После приведения подобных получаем систему уравнений:

$$
\sum_{j=1}^{n} \frac{\partial z_{j}}{\partial x_{i}}\left(x_{j}+\frac{\partial \psi}{\partial z_{j}}\right)=0
$$


Л. Л. Рыскина, Л. А. Жидова. Дифференциальное уравнение типа Клеро в частных производных со степенной функцией

Общее решение исходного уравнения (1) получается, когда $\frac{\partial z_{j}}{\partial x_{i}} \equiv 0$, $i, j=1,2, \ldots, n$, следовательно, все $z_{j}=C_{j}$, где $C_{j}$ - произвольные постоянные. Это согласуется с системой уравнений (5). Таким образом, общее решение уравнения (1) запишем в виде:

$$
y\left(x_{1}, x_{2}, \ldots, x_{n}\right)=\sum_{i=1}^{n} C_{i} x_{i}+\psi\left(C_{1}, C_{2}, \ldots, C_{n}\right) .
$$

Если $\frac{\partial z_{j}}{\partial x_{i}} \neq 0$ и матрица $\left\|\frac{\partial z_{j}}{\partial x_{i}}\right\|$ - невырожденная, тогда выражение в круглых скобках системы (5) обращается в ноль:

$$
x_{i}+\frac{\partial \psi\left(z_{1}, z_{2}, \ldots, z_{n}\right)}{\partial z_{i}}=0, \quad i=1,2, \ldots, n .
$$

Если система уравнений (7) имеет вещественные решения и величины $z_{i}$ выражаются как функции от аргументов $x_{1}, x_{2}, \ldots, x_{n}$, то уравнение (1) имеет особое решение:

$$
y=\sum_{i=1}^{n} x_{i} z_{i}(x)+\psi\left(z_{1}(x), z_{2}(x), \ldots ., z_{n}(x)\right) .
$$

Здесь для краткости использованы следующие обозначения:

$$
z_{i}(x)=z_{i}\left(x_{1}, x_{2}, \ldots, x_{n}\right), \quad i=1,2, \ldots, n .
$$

\section{2 Поиск особых решений}

Актуальность поиска особых решений уравнений типа Клеро в частных производных, отмечалась в недавних работах [7; 8]. В своей работе поиск особых решений будем проводить для функции $\psi\left(z_{1}, z_{2}, \ldots, z_{n}\right)$ специального вида.

Предположим, что функцию $\psi\left(z_{1}, z_{2}, \ldots, z_{n}\right)$ можно представить в виде произведения функций: $z_{1} \cdot z_{2} \cdot \ldots \cdot z_{n}$ в произвольной степени $\alpha$. Для начала рассмотрим частные случаи выбора $n=2,3,4$, а затем воспользуемся методом математической индукции и обобщим полученные результаты для произвольного $n \in \mathbb{N}$.

\section{1 Поиск особых решений для функции $\psi$ в виде произведения двух сомножителей}

Предположим, что функцию $\psi$ можно представить в виде произведения двух сомножителей: $z_{1} \cdot z_{2}$

$$
\psi\left(z_{1}, z_{2}\right)=\beta\left(z_{1} \cdot z_{2}\right)^{\alpha},
$$

где $\alpha, \beta-$ произвольные ненулевые вещественные числа. Система (7) примет вид: 


$$
\left\{\begin{array}{l}
x_{1}+\alpha \beta \cdot z_{2}\left(z_{1} \cdot z_{2}\right)^{\alpha-1}=0, \\
x_{2}+\alpha \beta \cdot z_{1}\left(z_{1} \cdot z_{2}\right)^{\alpha-1}=0 .
\end{array}\right.
$$

Выражаем из системы (11) произведение $z_{1} \cdot z_{2}$

$$
z_{1} \cdot z_{2}=\left((-1)^{2} \frac{x_{1} \cdot x_{2}}{(\alpha \beta)^{2}}\right)^{\frac{1}{2 \alpha-1}} .
$$

Так же из системы (11) можно найти произведение $x_{i} \cdot z_{i}, i=1,2$ :

$$
x_{1} \cdot z_{1}=x_{2} \cdot z_{2}=-\alpha \beta\left(z_{1} \cdot z_{2}\right)^{\alpha} \text {. }
$$

Подставляем (13) в выражение (8), получаем:

$$
y=(1-2 \alpha) \beta\left((-1)^{2} \frac{x_{1} \cdot x_{2}}{(\alpha \beta)^{2}}\right)^{\frac{\alpha}{2 \alpha-1}}, \quad \alpha \neq \frac{1}{2} .
$$

Выражение (14) является особым решением уравнения (1) для случая $n=2$. Данный результат является известным и описан в литературе [9].

\section{2 Поиск особых решений для функции $\psi$ в виде произведения} трех сомножителей

Предположим, что функцию $\psi$ можно представить в виде произведения трех сомножителей: $z_{1} \cdot z_{2} \cdot z_{3}$ :

$$
\psi\left(z_{1}, z_{2}, z_{3}\right)=\beta\left(z_{1} \cdot z_{2} \cdot z_{3}\right)^{\alpha} .
$$

Система (7) примет вид:

$$
\left\{\begin{array}{l}
x_{1}+\alpha \beta \cdot z_{2} \cdot z_{3}\left(z_{1} \cdot z_{2} \cdot z_{3}\right)^{\alpha-1}=0 \\
x_{2}+\alpha \beta \cdot z_{1} \cdot z_{3}\left(z_{1} \cdot z_{2} \cdot z_{3}\right)^{\alpha-1}=0 \\
x_{3}+\alpha \beta \cdot z_{1} \cdot z_{2}\left(z_{1} \cdot z_{2} \cdot z_{3}\right)^{\alpha-1}=0
\end{array}\right.
$$

Выражаем из системы (16) произведение $z_{1} \cdot z_{2} \cdot z_{3}$

$$
z_{1} \cdot z_{2} \cdot z_{3}=\left((-1)^{3} \frac{x_{1} \cdot x_{2} \cdot x_{3}}{(\alpha \beta)^{3}}\right)^{\frac{1}{3 \alpha-1}}
$$

Так же из системы (16) можно найти произведение $x_{i} \cdot z_{i}, i=1,2,3$ :

$$
x_{1} \cdot z_{1}=x_{2} \cdot z_{2}=x_{3} \cdot z_{3}=-\alpha \beta\left(z_{1} \cdot z_{2} \cdot z_{3}\right)^{\alpha}
$$

Подставляем (18) в выражение (8), получаем:

$$
y=(1-3 \alpha) \beta\left((-1)^{3} \frac{x_{1} \cdot x_{2} \cdot x_{3}}{(\alpha \beta)^{3}}\right)^{\frac{\alpha}{3 \alpha-1}}, \quad \alpha \neq \frac{1}{3} .
$$


Л. Л. Рыскина, Л. А. Жидова. Дифференциальное уравнение типа Клеро в частных производных со степенной функцией

Выражение (19) является особым решением уравнения (1) для случая $n=3$. Полученный результат ранее не был описан в литературе и является новым.

\section{3 Поиск особых решений для функции $\psi$ в виде произведения четырех сомножителей}

Предположим, что функцию $\psi$ можно представить в виде произведения четырех сомножителей: $z_{1} \cdot z_{2} \cdot z_{3} \cdot z_{4}$

$$
\psi\left(z_{1}, z_{2}, z_{3}, z_{4}\right)=\beta\left(z_{1} \cdot z_{2} \cdot z_{3} \cdot z_{4}\right)^{\alpha} .
$$

Система (7) примет вид:

$$
\left\{\begin{array}{l}
x_{1}+\alpha \beta \cdot z_{2} \cdot z_{3} \cdot z_{4}\left(z_{1} \cdot z_{2} \cdot z_{3} \cdot z_{4}\right)^{\alpha-1}=0, \\
x_{2}+\alpha \beta \cdot z_{1} \cdot z_{3} \cdot z_{4}\left(z_{1} \cdot z_{2} \cdot z_{3} \cdot z_{4}\right)^{\alpha-1}=0, \\
x_{3}+\alpha \beta \cdot z_{1} \cdot z_{2} \cdot z_{4}\left(z_{1} \cdot z_{2} \cdot z_{3} \cdot z_{4}\right)^{\alpha-1}=0, \\
x_{4}+\alpha \beta \cdot z_{1} \cdot z_{2} \cdot z_{3}\left(z_{1} \cdot z_{2} \cdot z_{3} \cdot z_{4}\right)^{\alpha-1}=0 .
\end{array}\right.
$$

Выражаем из системы (21) произведение $z_{1} \cdot z_{2} \cdot z_{3} \cdot z_{4}$

$$
z_{1} \cdot z_{2} \cdot z_{3} \cdot z_{4}=\left((-1)^{4} \frac{x_{1} \cdot x_{2} \cdot x_{3} \cdot x_{4}}{(\alpha \beta)^{4}}\right)^{\frac{1}{4 \alpha-1}} .
$$

Так же из системы (21) можно найти произведение $x_{i} \cdot z_{i}, i=1,2,3,4$ :

$$
x_{1} \cdot z_{1}=x_{2} \cdot z_{2}=x_{3} \cdot z_{3}=x_{4} \cdot z_{4}=-\alpha \beta\left(z_{1} \cdot z_{2} \cdot z_{3} \cdot z_{4}\right)^{\alpha} .
$$

Подставляем (23) в выражение (8), получаем:

$$
y=(1-4 \alpha) \beta\left((-1)^{4} \frac{x_{1} \cdot x_{2} \cdot x_{3} \cdot x_{4}}{(\alpha \beta)^{4}}\right)^{\frac{\alpha}{4 \alpha-1}}, \quad \alpha \neq \frac{1}{4} .
$$

Выражение (24) является особым решением уравнения (1) для случая $n=4$. Полученный результат также ранее не был описан в литературе и является новым.

\section{4 Обобщение полученных результатов для функции $\psi$ в виде} произведения $\boldsymbol{n}$ сомножителей

Для обобщения полученных результатов, рассмотрим случай, когда функцию $\psi$ можно представить в виде произведения $n$-сомножителей:

$$
\psi\left(z_{1}, z_{2}, \ldots, z_{n}\right)=\beta\left(z_{1} \cdot z_{2} \cdot \ldots \cdot z_{n}\right)^{\alpha} .
$$

Система (7) примет вид: 


$$
\left\{\begin{array}{c}
x_{1}+\alpha \beta \cdot z_{2} \cdot z_{3} \cdot \ldots \cdot z_{n}\left(z_{1} \cdot z_{2} \cdot \ldots \cdot z_{n}\right)^{\alpha-1}=0 \\
x_{2}+\alpha \beta \cdot z_{1} \cdot z_{3} \cdot \ldots \cdot z_{n}\left(z_{1} \cdot z_{2} \cdot \ldots \cdot z_{n}\right)^{\alpha-1}=0 \\
\ldots \\
x_{n}+\alpha \beta \cdot z_{1} \cdot z_{2} \cdot \ldots \cdot z_{n-1}\left(z_{1} \cdot z_{2} \cdot \ldots \cdot z_{n}\right)^{\alpha-1}=0 .
\end{array}\right.
$$

Выражаем из системы (26) произведение $z_{1} \cdot z_{2} \cdot \ldots \cdot z_{n}$

$$
z_{1} \cdot z_{2} \cdot \ldots \cdot z_{n}=\left((-1)^{n} \frac{x_{1} \cdot x_{2} \cdot \ldots \cdot x_{n}}{(\alpha \beta)^{n}}\right)^{\frac{1}{n \alpha-1}} .
$$

Так же из системы (26) можно найти произведение $x_{i} \cdot z_{i}, i=1,2, \ldots, n$ :

$$
x_{1} \cdot z_{1}=x_{2} \cdot z_{2}=\ldots=x_{n} \cdot z_{n}=-\alpha \beta\left(z_{1} \cdot z_{2} \cdot \ldots \cdot z_{n}\right)^{\alpha} .
$$

Подставляем (28) в выражение (8), получаем:

$$
y=(1-n \alpha) \beta\left((-1)^{n} \frac{x_{1} \cdot x_{2} \cdot \ldots \cdot x_{n}}{(\alpha \beta)^{n}}\right)^{\frac{\alpha}{n \alpha-1}}, \quad \alpha \neq \frac{1}{n} .
$$

Выражение (29) является особым решением уравнения (1) для произвольного случая $n \in \mathbb{N}$. Формула (29) является центральным результатом данной работы и ранее не рассматривалась в литературе.

\section{Заключение}

Полученное в данной работе особое решение (29) уравнение типа Клеро в частных производных (1), со специальной правой частью, представленное выражением (25), по нашему мнению, можно рассматривать как новый результат в теории дифференциальных уравнений в частных производных. Заметим, что из найденного особого решения (29), при $n=2$, следует ранее полученное особое решение в работе [9]. Из этого можно судить о правильности полученного результата и корректности сделанного обобщения.

Об актуальности полученных в настоящей работе результатов для теории дифференциальных уравнений в частных производных можно судить по большому количеству статей, посвященных нахождению особых решений уравнений типа Клеро (см., например, [7-10]). Однако поиск особых решений для конкретных функций остается малоразработанным и представляет собой перспективное направление для дальнейших исследований.

\section{Литература}

1. Зайцев В. Ф., Полянин А. Д. Справочник по обыкновенным дифференциальным уравнениям. М.: Физматлит, 2002. 256 с.

2. Степанов В. В. Курс дифференциальных уравнений. М.: Физматлит, 1965. $512 \mathrm{c}$. 
Л. Л. Рыскина, Л. А. Жидова. Дифференциальное уравнение типа Клеро в частных производных со степенной функцией

3. Эльсгольц Л. Э. Дифференциальные уравнения и вариационное исчисление. М.: Наука, 1969. 424 с.

4. Зайцев В. Ф., Полянин А. Д. Справочник по дифференциальным уравнениям в частных производных первого порядка. М.: Физматлит, 2003. 416 с.

5. Камке Э. Справочник по дифференциальным уравнениям в частных производных первого порядка. М.: Наука, 1966. 260 с.

6. Курант Р. Уравнения с частными производными. М.: Мир, 1964. 830 с.

7. Lavrov P. M., Merzlikin B. S. Legendre Transformations and Clairaut-Type Equations // Physics Letters. 2016. V. 756. Pp. 188-193.

8. Lavrov P. M., Merzlikin B. S. Loop expansion of the average effective action in the functional renormalization group approach // Phys. Rev. 2015. Vol. 92, No. 8. [085038].

9. Жидова Л. А., Зырянова О. В., Холмухаммад Ф. Дифференциальные уравнения в профессиональной подготовке учителя математики // Вестник ТГПУ. 2017. № 1 (178). С. 75-78.

10. Рахмелевич И. В. О решениях многомерного уравнения Клеро с мультиоднородной функцией от производных // Известия Саратовского университета. Новая серия. Сер. Математика, механика, информатика. 2014. Т. 14, № 4-1. C. 374-381.

\section{A CLAIRAUT-TYPE DIFFERENTIAL EQUATION IN PARTIAL DERIVATIVES WITH A POWER FUNCTION}

Liliya L. Ryskina

Cand. Sci. (Phys. and Math.), A/Prof.,

Tomsk State Pedagogical University

60 Kievskaya St., Tomsk 634061, Russia

E-mail: ryskina@tspu.edu.ru

Lyubov A. Zhidova

Cand. Sci. (Education), A/Prof.,

Tomsk State Pedagogical University

60 Kievskaya St., Tomsk 634061, Russia

E-mail: gidovala@yandex.ru

The article is aimed at the study of Clairaut-type differential equations in partial derivatives of the first order with a special right-hand side. Finding a general solution for an ordinary Clairaut differential equation is not difficult, and it is described in detail in courses on the theory of ordinary differential equations. In addition to the general solution, which involves a family of integral lines, there is a special solution for an ordinary Clairaut differential equation, represented by the envelope of this family. Within the framework of the theory of differential equations in partial derivatives there are Clairaut-type differential equations, which are multidimensional generalizations of an ordinary Clairaut differential equation. Note that for a Clairaut-type partial differential equation there is no always a special solution. The article is devoted to the problem of the description of a particular solution for Clairaut-type equations, which right side has the form of a power function of the product of $\mathrm{n}$ factors.

Keywords: partial differential equations; Clairaut-type differential equations; singular solutions; power function. 


\section{References}

1. Zaitsev V. F., Polyanin A. D. Spravochnik po obyknovennym differentsial'nym uravneniyam [Reference Book on Ordinary Differential Equations]. Moscow: Fizmatlit Publ., 2002. 256 p.

2. Stepanov V. V. Kurs differentsialnykh uravnenii [A Course in Differential Equations]. Moscow: Fizmatlit Publ., 1965. 512 p.

3. Elsgolts L. E. Differentsialnye uravneniya $i$ variatsionnoe ischislenie [Differential Equations and Variational Calculus]. Moscow: Nauka Publ., 1969. 424 p.

4. Zaitsev V. F., Polyanin A. D. Spravochnik po differentsialnym uravneniyam v chastnykh proizvodnykh pervogo poryadka [Reference Book on Differential Equations in Partial Derivatives of the First Order]. Moscow: Fizmatlit Publ., 2003. 416 p.

5. Kamke E. Spravochnik po differentsialnym uravneniyam v chastnykh proizvodnykh pervogo poryadka [Reference Book on Differential Equations in First-Order Partial Derivatives]. Moscow: Nauka Publ., 1966. 260 p. (transl. from German)

6. Courant R., and Hilbert D. Methods of Mathematical Physics. V. 2. Partial Differential Equations. New York: Interscience, 1962. 830 p.

7. Lavrov P. M., Merzlikin B. S. Legendre Transformations and Clairaut-Type Equations. Physics Letters. 2016. V. 756. P. 188-193.

8. Lavrov P. M., Merzlikin B. S. Loop Expansion of the Average Effective Action in the Functional Renormalization Group Approach. Phys. Rev. 2015. V. 92. No. 8. [085038].

9. Zhidova L. A., Zyryanova O. V., Kholmukhammad F. Differentsialnye uravneniya $\mathrm{v}$ professionalnoi podgotovke uchitelya matematiki [Differential Equations in the Professional Training of Mathematics Teachers]. TSPU Bulletin. 2017. No. 1 (178). Pp. 75-78.

10. Rakhmelevich I. V. O resheniyakh mnogomernogo uravneniya Klero s multiodnorodnoi funktsiei ot proizvodnykh [On the Solutions of Multi-Dimensional Clairaut Equation with a Multi-Homogeneous Function of Derivatives]. Izvestiya Saratovskogo universiteta. Novaya seriya. Ser. Matematika, mekhanika, informatika. 2014. V. 14. No. 4-1. Pp. 374-381. 